\title{
Ab initio study of the structure and energetic of pyridine dimers
}

\author{
Vladimir Sladek, Michal Ilčin \\ Institute of Physical Chemistry and Chemical Physics, Slovak Technical University, \\ SK-81237 Bratislava, Slovakia \\ vladimir_sladek@stuba.sk
}

\begin{abstract}
Strong correlation of stabilization energies of $\pi$-stacked pyridine and fluorinated pyridine dimers with various relative orientations is presented. Four possible orientations of the monomers were considered. A SAPT decomposition of the interaction energies is presented and briefly discussed. The dominant electrostatic contribution to the stabilization energy is found in some dimers and its possible origin is addressed in the discussion. An outline of possible future studies is introduced.
\end{abstract}

Keywords: $\pi$ - stacking, weak interaction, fluorine substitution, molecular recognition

\section{Introduction}

Intermolecular interactions account for the properties of the system in many areas of chemistry. Interactions of aromatic molecules ( $\pi-\pi$ interactions) play a significant role in the determination of the crystal structures and molecular recognition in both biological and artificial systems (Hunter et al. 2001, Meyer et al. 2003).

Many studies on interactions of six membered carbon rings both substituted and non-substituted were published (e.g. Tsuzuki et al. 2006, Pitoňák et al. 2008, Gung et al. 2006). Predominantly, the effects of relative orientation (e.g. stack, T, etc.) and the substitution effect on the interaction energy and stability of the super systems were described.

Both six and five membered rings with hetero atoms analogously play an important role as the six membered carbon rings in many types of systems (Berg et al. 2010). Examples of such systems may be interactions of DNA base pairs or aromatic side chains interaction in proteins and other bio polymers as well as application in material or nano science, to name just a few (Lee at al. 2007).

To our knowledge no works on the substitution effect and the effect of relative orientation in six membered rings with hetero atoms have been published yet. Following this and the fact that the methods employed are routinely used in such type of studies, the aims of this work can be summarized as follows; to investigate the effect of substitution (fluorination) and the relative orientation of pyridine molecules in $\pi-\pi$ sandwich interactions on the stability of the dimer. We restrict ourselves to the $\pi / \pi$ sandwich structures as these are of principal importance in biological systems (Hunter et al. 2001, Meyer et al. 2003). Amongst all the fluorination possibilities, only the pentafluoropyridine will be considered. A basic SAPT (Symmetry Adapted Per- turbation Theory) decomposition of the interaction energy will be performed on some of the systems and the importance of selected contributions will be presented. The pyridine and pentafluoropyridine systems have to be considered as a model with respect to the possible applications mentioned in the latter.

\section{Computational details}

The electronic singlet ground state structures of the studied molecules were optimized at the MP2 level of theory (Möller, Plesset second order perturbation theory, 1934). The employed basis set for all atoms $(\mathrm{C}, \mathrm{N}, \mathrm{F})$ but hydrogen was of the aug-cc-pVDZ quality and for hydrogen the non augmented ccpVDZ was used (Dunning 1989, Woon 1993). This combination will be denoted as (aug)-cc-pVDZ. All the bases are implemented in the Gaussian03 basis set library (Frisch et al. 2003). The energy cut-off was of $10^{-4} \mathrm{~kJ} \mathrm{~mol}^{-1}$ and the final root mean square energy gradient was under $0.005 \mathrm{~kJ} \mathrm{~mol}^{-1} \AA^{-1}$.

Single point calculations with the optimized geometries in aug-cc-pVTZ (atoms C, N, F) and ccpVTZ (for H atom), abbreviated as (aug)-cc-pVTZ, were used for the evaluation of the interaction energies following the established scheme of Xantheas (Xantheas 1996). The employed Dunning's basis sets are correlation consistent, which justifies for the use of higher cardinality bases for the calculation of the interaction energy. All MP2 calculations through this work were in full active space (all electrons correlated). All the quantum chemical calculations were carried out using the Gaussian03 package (Frisch et al. 2003).

The SAPT (Bukowski et. al. 2008) calculations were done in 6-311G(d,p) basis set (Krishnan et al. 1980). Only selected contributions are depicted in this work. 


\section{Results and discussion}

\section{Note on the used method - comparison with other theoretical approaches on published $\pi-\pi$ sandwich structures}

The validity of the selected method for the evaluation of the interaction energies was tested on reference systems from already published works (e.g. Tsuzuki et al. 2006, Pitoňák et al. 2008, Gung et al. 2006). Benzene - benzene, benzene - hexafluorobenzene and hexafluorobenzene - hexafluorobenzene dimers in $\pi$-stacked (sandwich) structures were chosen. These were recomputed for this work for the sake of justification of our approach.

Tsuzuki et al. (2006) gives the BSSE free MP2/augcc-pVTZ interaction energy of benzene - hexafluorobenzene in sandwich structure as $-36.62 \mathrm{~kJ} \mathrm{~mol}^{-1}$ and our approach provides $-37.80 \mathrm{~kJ} \mathrm{~mol}^{-1}$, i.e. a deviance of $3.2 \%$. However, in the same paper the CCSD $(\mathrm{T})$ calculation at the basis set limit gives only $-9.94 \mathrm{~kJ} \mathrm{~mol}^{-1}$. The active space configuration of Tsuzuki is not specifically discussed. All results in our work were calculated in the full active space. On the other hand, Gung et al. (2006) provides the BSSE free MP2(full)/6-31+G** interaction energy for benzene - hexafluorobenzene as $-21.42 \mathrm{~kJ} \mathrm{~mol}^{-1}$. Con- sidering the basis set dependence of the results, our value of the interaction energy is in the closest agreement with the value of Tsuzuki et al. (2006).

Pitoňák et al. (2008) report the CBS (Complete Basis Set) extrapolated CCSD(T)/aug-cc-pV[Q5]Z stabilization energy of the benzene - benzene dimer in sandwich structure as $-7.02 \mathrm{~kJ} \mathrm{~mol}^{-1}$, whereas our approach gives $-12.47 \mathrm{~kJ} \mathrm{~mol}^{-1}$. Considering the difference in the ability of MP2 and CCSD(T) to describe the interaction energy correctly (see Tsuzuki et al. 2006, who have a 3.7 times larger interaction energy for MP2 than for $\operatorname{CCSD}(\mathrm{T})$ ) it is hard to conclude on the quality of our result for benzenebenzene dimer. It is also known that for such type of dimers, the non-covalent weak interactions tend to have oscillatory behavior (in magnitude) with respect to the order of perturbation in the MøllerPlesset approach (Tsuzuki et al. 2000). MP2 tends to overestimate the interaction energy in magnitude, whereas CCSD and MP3 calculations usually underestimate these energies (Pitoňák et al. 2008a). Hence, the discrepancy of the coupled cluster value of Pitoňák et al. (2008) and our MP2 result.

In case of the hexafluorobenzene - hexafluorobenzene in sandwich structure a value of $-17.85 \mathrm{~kJ} \mathrm{~mol}^{-1}$ is obtained by our approach.

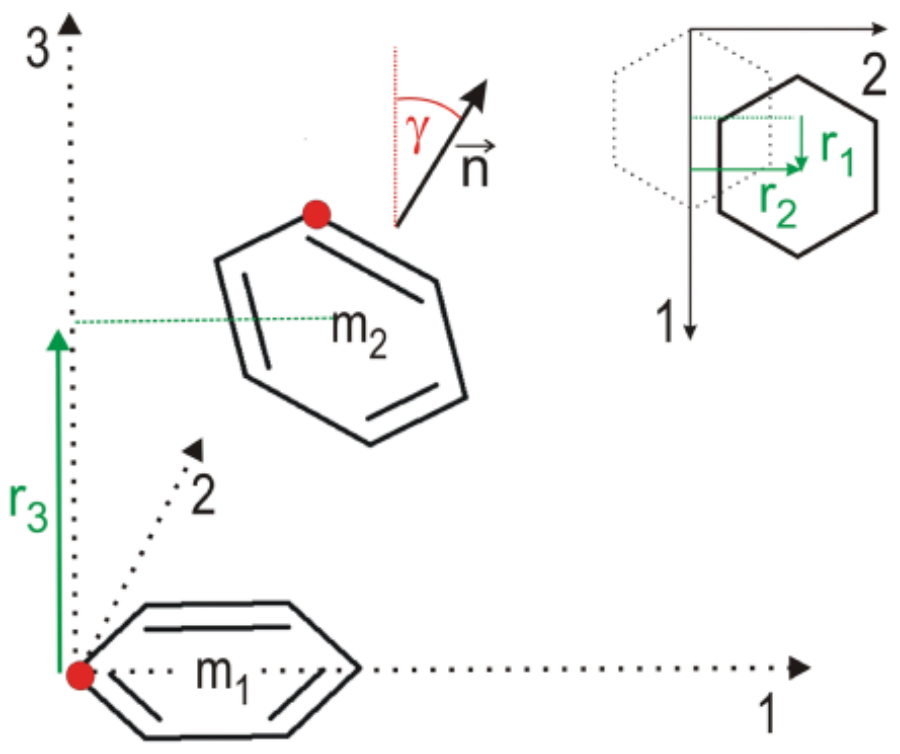

Fig. 1. Definition of the coordinate system used in this work. It is assumed that one ring is in the 1-2 plane (e.g. ring 1). The origin of the coordinate system is set on the nitrogen on ring 1 (nitrogen is indicated by the red dot). The perpendicular distance between the geometric centers of the two rings $\left(m_{1}\right.$ and $m_{2}$ in the monomer 1 and 2, respectively) is $r_{3}$ (corresponds to the $x_{3}$ coordinate of the centre $m_{2}$ since $m_{1}$ is in the 1-2 plane). The inset picture defines coordinates $r_{1}$ and $r_{2}$ which describe the relative shift of the second ring in the 1-2 plane $\left(r_{1}\right.$ corresponds to the $x_{1}\left(m_{2}\right)-x_{1}\left(m_{1}\right)$ distance and $r_{2}$ is the $x_{2}$ coordinate of the centre $m_{2}$ ). The relative tilt of the second ring, $\gamma$, is measured as the angle between the normal vectors of the planes of the two rings. The relative orientation of the two nitrogen atoms is given by the dihedral angle $\delta$, defined by these four subsequent points: $\mathrm{N}_{1}, m_{1}, m_{2}, \mathrm{~N}_{2}\left(\mathrm{~N}_{1(2)}\right.$ being the nitrogen atoms in ring 1 and 2). 


\section{Pyridine and substituted pyridine dimers}

One of the aims of this work is to investigate the effect of the relative orientation of the two rings on the strength of interaction. We adopt the following nomenclature to facilitate the description of the relative orientation of the nitrogen atoms (more exactly described by the dihedral angle $\delta$ ). Ortho, abbreviated as $o$, correspond to the starting geometry with $\delta=60^{\circ}$. Analogically, meta $\left(m, \delta=120^{\circ}\right)$, para $\left(p, \delta=180^{\circ}\right)$ and parallel $\left(p l, \delta=0^{\circ}\right)$.

The geometry optimization in all cases started from a perfectly coplanar sandwich structure in the highest possible symmetry (i.e. $C_{\mathrm{s}}$ for $p l$ ) symmetry. We propose the following scheme (see Figure 1) to describe the relative position of the two interacting rings. This allows the description of the distance of the geometric centers, the relative rotation and the declination from coplanarity.

In the introduction part a brief comparison of the methodologies used in comparable studies is presented. The reasonably good correspondence of the interaction energies justifies for our choice of methodology. Table 1 contains the BSSE corrected interaction energies (or stabilization energies). From the first glance it is evident that these do strongly depend on the relative orientation $(p l, o, m, p)$ of the two monomers within one combination of the monomers (i.e. HP-PH, HP-PF or FP-PF). In the case of the HP-PH dimers, the $o$ and $m$ orientations exhibit huge stabilization energies compared to the $p l$ an $\mathrm{d} p$ orientations. This is mainly due to the availability of both $\mathrm{N}$ atoms to interact with hydrogen atoms from the other monomer when in $o$ or $m$ orientation. In the case of the $p$ and $p l$ orientation such interaction is not possible. It is important to note, that the $o$ orientation caused a considerable drift away from the starting $\delta=60^{\circ}$ and it converged to $\delta=100^{\circ}$. This is comparable to the $m$ orientation. The weakest stabilization is in the $p l$ orientation. Here the direct alignment of the $\mathrm{N}$ atoms (the $C_{\mathrm{s}}$ symmetry is held almost perfectly) forces the two monomers to the biggest separation, $r_{3}=3.47 \AA$. This is not the case in the $p$ orientation, however, this cannot be stabilized by two $\mathrm{N}-\mathrm{H}$ interactions either.

In the case of the mixed HP-PF dimers, both the $p l$ and $p$ structures are displaced along the first axis (see Figure 1). Therefore the initial symmetry is broken. In the case of the $o$ structure the alignment of the molecules is such that the nitrogen atom from the fluorinated monomer is in the closest proximity to the hydrogen on the $\mathrm{C}_{1}$ carbon (next to the $\mathrm{N}$ atom) of the non-substituted pyridine (the distance is approx. $3.05 \AA$ ). The nitrogen of the non-substituted molecule is in the proximity of the $\mathrm{C}_{2}$ carbon (second from the $\mathrm{N}$ atom) of the fluorinated pyridine (the distance is approx. 3.01 $\AA$ ). In this context the Merz-Kollman ESP charges (Singh et al. 1984, Besler et al. 1990) of the monomers are evaluated. The charges are presented in Table 2.

The FP-PF dimers exhibit somewhat different behavior than the mixed HP-PF dimers. The analogy with the HP-PH dimers structures is rather obvious. This is mainly due the fact, that in these two cases the partial charges are identical at both monomers.

Tab. 1. The coordinates $r_{1}, r_{2}, r_{3}, \gamma$ and $\delta$ of the MP2/(aug)-cc-pVDZ optimized geometries of the weakly bound dimers. Based on these, the BSSE corrected interaction energies $\Delta E$ are computed at the MP2/(aug)-cc-pVTZ level of theory. HP-PH stands for the pyridine-pyridine dimer, HP-PF for the pyridine-pentafluoropyridine and FP-PF for pentafluoropyridine dimer.

\begin{tabular}{|c|c|c|c|c|c|c|}
\hline orientation & $\mathbf{r}_{1} / \AA$ & $\mathbf{r}_{2} / \AA$ & $\mathbf{r}_{3} / \AA$ & $\gamma / \operatorname{deg}$ & $\delta / \operatorname{deg}$ & $\Delta \mathrm{E} / \mathrm{kJ} \mathrm{mol}^{-1}$ \\
\hline \multicolumn{7}{|c|}{ HP-PH } \\
\hline$p l$ & 0.00 & 0.01 & 3.47 & 0.4 & 0 & -11.66 \\
\hline$o$ & 0.94 & 0.92 & 3.25 & 4.7 & 100 & -1605.56 \\
\hline$m$ & 1.11 & 0.60 & 3.15 & 2.4 & 123 & -1602.38 \\
\hline$p$ & 1.26 & 0.01 & 3.17 & 0.0 & 180 & -19.92 \\
\hline \multicolumn{7}{|c|}{ HP-PF } \\
\hline$p l$ & -0.97 & 0.01 & 3.13 & 3.9 & 0 & -820.74 \\
\hline$o$ & -0.88 & -0.62 & 3.03 & 2.6 & 75 & -823.05 \\
\hline$m$ & -0.80 & -0.73 & 3.03 & 0.9 & 102 & \\
\hline$p$ & 0.81 & 0.00 & 3.09 & 1.2 & 180 & -761.54 \\
\hline \multicolumn{7}{|c|}{ FP-PF } \\
\hline$p l$ & 0.06 & 0.01 & 3.21 & 2.1 & 0 & -13.71 \\
\hline$o$ & 0.74 & -0.97 & 3.08 & 0.6 & 69 & -30.49 \\
\hline$m$ & 0.88 & 0.37 & 3.01 & 1.1 & 136 & -20.48 \\
\hline$p$ & 0.93 & 0.01 & 3.04 & 0.0 & 180 & -19.29 \\
\hline
\end{tabular}


Tab. 2. The ESP (Merz-Kollman) fitted partial charges based on the MP2/(aug)-cc-pVTZ densities of the monomers pyridine (HP) and pentafluoropyridine (FP). $\mathrm{C}_{1}$ is the carbon next to nitrogen. Then $\mathrm{C}_{2}$ is the second one and $\mathrm{C}_{3}$ the third one from nitrogen. $\mathrm{X}_{1(2,3)}$ are the corresponding substituents $(\mathrm{H}$ on pyridine, $\mathrm{F}$ on pentafluoropyridine) on carbons $\mathrm{C}_{1(2,3)}$ respectively. The values separated by "/" correspond to the symmetrically positioned atoms. The default Merz-Kollman radii were used.

\begin{tabular}{|c|c|c|c|c|}
\hline & $\begin{array}{c}\mathbf{C}_{1} \\
\left(\mathbf{X}_{1}\right)\end{array}$ & $\begin{array}{c}\mathbf{C}_{2} \\
\left(\mathbf{X}_{2}\right)\end{array}$ & $\begin{array}{c}\mathbf{C}_{3} \\
\left(\mathbf{X}_{3}\right)\end{array}$ & $\mathbf{N}$ \\
\hline HP & $\begin{array}{c}0.49 / 0.49 \\
(0.01 / 0.01)\end{array}$ & $\begin{array}{c}-0.52 /-0.52 \\
(0.18 / 0.18)\end{array}$ & $\begin{array}{c}0.27 \\
(0.06)\end{array}$ & -0.67 \\
\hline FP & $\begin{array}{c}0.43 / 0.44 \\
(-0.16 /-0.16)\end{array}$ & $\begin{array}{c}0.01 / 0.00 \\
(-0.11 /-0.11)\end{array}$ & $\begin{array}{c}0.25 \\
(-0.12)\end{array}$ & -0.47 \\
\hline
\end{tabular}

Again the $p l$ structure is the most weakly stabilized and the deflection from the starting geometry is smallest. The molecules stay in almost perfect alignment. Again the separation of the monomers is the biggest, approx. $3.21 \AA$. On the other hand, in the case of the FP-PF dimers, the stabilization of the structure by $\mathrm{N}-\mathrm{H}$ interactions is not possible. Hence, the $o$ and $m$ structures do not exhibit such a dominant increase in the interaction energy as is in the HP-PH dimers. This implies that even though the $r_{1}$ and $r_{2}$ coordinates in FP-PF and HP-PH dimers are comparable, the main difference in their structure is the dihedral angle $\delta$. This angle defines the relative orientation of the two monomers (see Figure 1). While in the HP-PH dimers the orientation of the monomers is in favor of the $\mathrm{N}-\mathrm{H}$ interaction, in the case of the FP-PF dimers their orientation is such that the distance between $\mathrm{F}$ atoms is maximized. Certainly no N-F stabilization can occur (at least due to the negative partial charges of both atoms).

The Merz-Kollman ESP charges (in a.u.) for selected dimers were calculated and collected in Table 3 . The
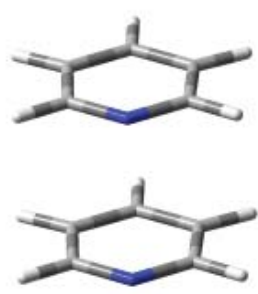

HP-PH-pl
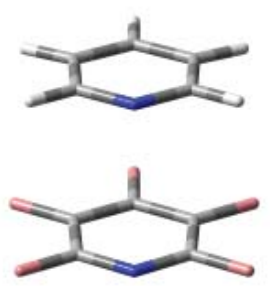

HP-PF- $p l$
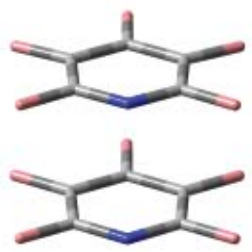

FP-PF- $p l$
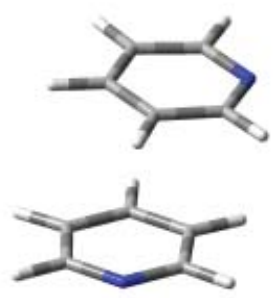

HP-PH-o
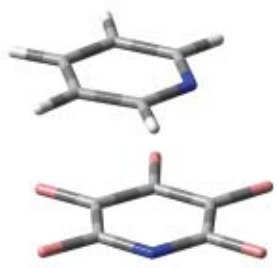

HP-PF-o

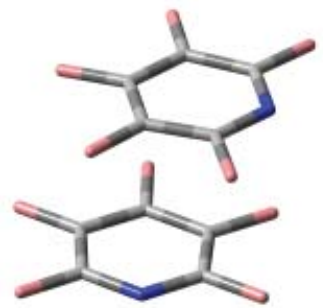

FP-PF-o
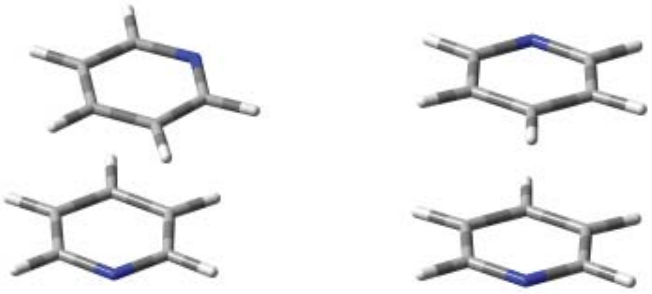

HP-PH- $m$

HP-PH- $p$

Fig. 2. The MP2/(aug)-cc-pVDZ optimized structures of the $\pi$ stacked dimers. 
partial charge distribution in free and interacting monomers may diverge; see the comparison of the data in Table 2 and 3. Evidently the charge on $\mathrm{C}_{3}$ in PF changes from 0.013 (and 0,002) to -0.77 when interacting in the "ortho" orientation.
Some figures of the dimer structures are collected in the Figure 2.

The SAPT decomposition of the stabilization energy was done for the two cases with the strongest and weakest interaction, i.e. НР-РН-o and HР-PH-pl,

Tab. 3. The ESP (Merz-Kollman) fitted partial charges based on the MP2/(aug)-cc-pVTZ densities of the dimers in $p l$ and $o$ orientations. $\mathrm{C}_{1}$ is the carbon next to nitrogen. Then $\mathrm{C}_{2}$ is the second one and $\mathrm{C}_{3}$ the third one from nitrogen. $\mathrm{X}_{1(2,3)}$ are the corresponding substituents $(\mathrm{H}$ on pyridine, $\mathrm{F}$ on pentafluorpyridine) on carbons $\mathrm{C}_{1(2,3)}$ respectively. The values separated by "/" correspond to the atoms in the same position, but in different molecules. In the case of the HP-PF dimers the values on HP are the first ones. The default Merz-Kollman radii were used.

\begin{tabular}{|c|c|c|c|c|}
\hline & $\begin{array}{c}\mathbf{C}_{1} \\
\left(\mathbf{X}_{1}\right)\end{array}$ & $\begin{array}{c}\mathbf{C}_{2} \\
\left(\mathbf{X}_{2}\right)\end{array}$ & $\begin{array}{c}\mathbf{C}_{3} \\
\left(\mathbf{X}_{3}\right) \\
\end{array}$ & $\mathbf{N}$ \\
\hline HP-PH-pl & $\begin{array}{c}0.44 / 0.42 \\
(0.03 / 0.04)\end{array}$ & $\begin{array}{c}-0.52 /-0.50 \\
(0.19 / 0.19)\end{array}$ & $\begin{array}{c}0.29 / 0.26 \\
(0.05 / 0.06)\end{array}$ & $-0.62 /-0.61$ \\
\hline НР-PH-o & $\begin{array}{c}0.44 / 0.51 \\
(0.03 / 0.02)\end{array}$ & $\begin{array}{c}-0.52 /-0.59 \\
(0.19 / 0.22)\end{array}$ & $\begin{array}{c}0.30 / 0.34 \\
(0.06 / 0.04)\end{array}$ & $-0.67 /-0.69$ \\
\hline HP-PF- $p l$ & $\begin{array}{c}0.47 / 0.51 \\
(0.03 /-0.17)\end{array}$ & $\begin{array}{l}-0.56 /-0.11 \\
(0.21 /-0.09)\end{array}$ & $\begin{array}{c}0.33 / 0.31 \\
(0.06 /-0.12)\end{array}$ & $-0.64 /-0.49$ \\
\hline HP-PF- $O$ & $\begin{array}{c}0.44 / 0.47 \\
(0.04 /-0.16)\end{array}$ & $\begin{array}{l}-0.48 /-0.03 \\
(0.18 /-0.09)\end{array}$ & $\begin{array}{c}0.27 / 0.22 \\
(0.07 /-0.11)\end{array}$ & $-0.63 /-0.49$ \\
\hline FP-PF- $p l$ & $\begin{array}{c}0.44 / 0.44 \\
(-0.15 /-0.15)\end{array}$ & $\begin{array}{c}-0.77 /-0.77 \\
(-0.08 /-0.08)\end{array}$ & $\begin{array}{c}0.29 / 0.29 \\
(-0.12 /-0.12)\end{array}$ & $-0.47 /-0.47$ \\
\hline FP-PF-o & $\begin{array}{c}0.41 / 0.46 \\
(-0.13 /-0.15) \\
\end{array}$ & $\begin{array}{c}-0.04 /-0.06 \\
(-0.09 /-0.09)\end{array}$ & $\begin{array}{c}0.28 / 0.25 \\
(-0.12 /-0.11)\end{array}$ & $-0.47 /-0.46$ \\
\hline
\end{tabular}

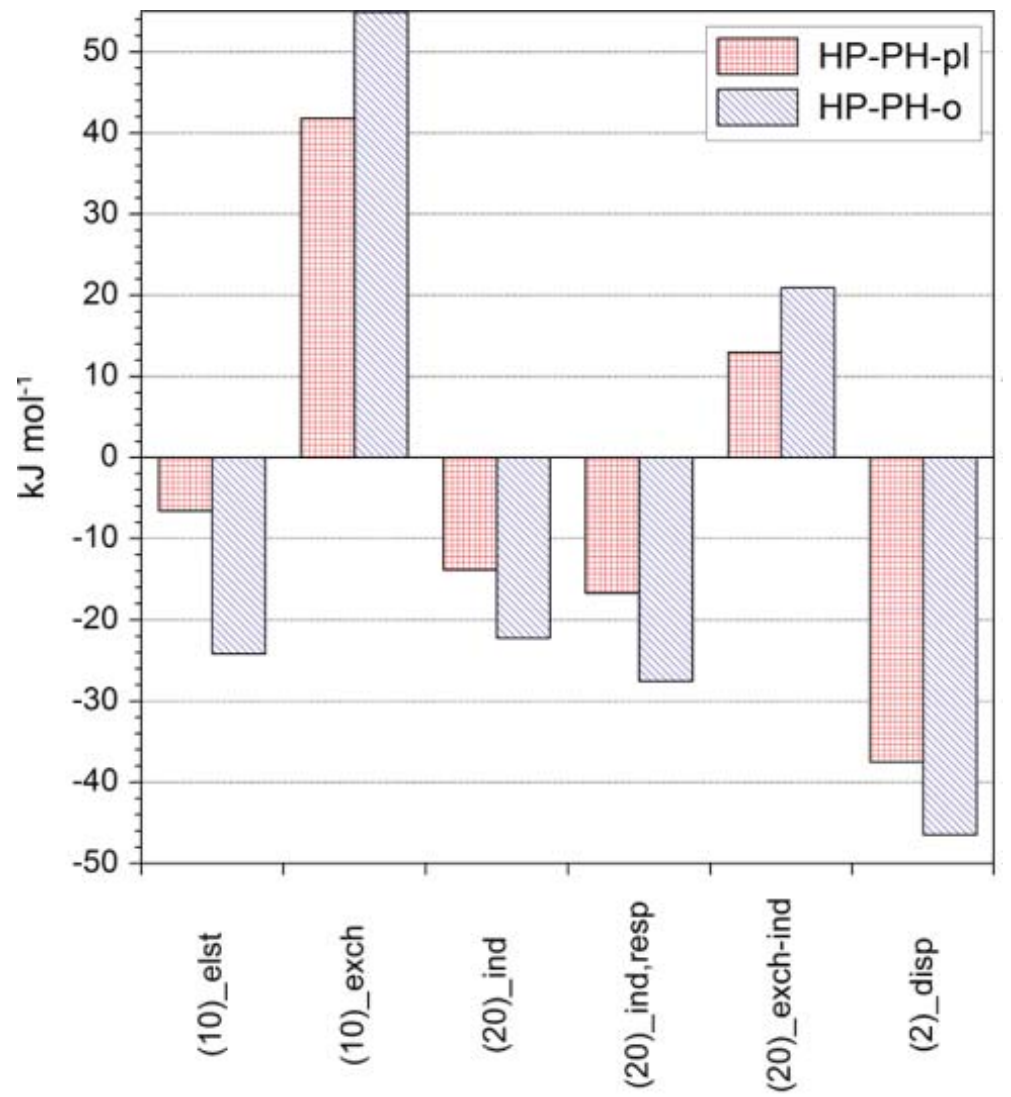

Fig. 3. The selected SAPT contributions to the stabilization energy for the cases with the strongest and the weakest interaction. (10)_elst is $E^{(10)}{ }_{\text {elst }},(10)$ exch is $E^{(10)}{ }_{\text {exch }},(20)$ ind is $E^{(20)}{ }_{\text {ind }},(20)$ ind, resp is $E^{(20)}$ ind, resp, (20)_exch-ind is $E_{\text {exch-ind }}^{(20)}$ and (2)_disp is $E_{\text {disp. }}^{(2)}$. 
respectively. As anticipated, the dominant difference between the HP-PH- $o$ an d HP-PH-pl dimers is in the electrostatic contribution. The $E^{(10)}$ elst of $\mathrm{HP}-\mathrm{PH}-o$ is 3.66 times larger than for HP-PH- $p l$ $\left(266 \%\right.$ change w.r.t. $E^{(10)}$ elst of HP-PH- $\left.p l\right)$. On the other hand, the exchange contribution $E^{(10)}$ exch does not vary so dramatically with respect to the orientation of the monomers. It is $41.8 \mathrm{~kJ} \mathrm{~mol}^{-1}$ for HP-PH- $p l$ and $54.9 \mathrm{~kJ} \mathrm{~mol}^{-1}$ for HP-PH-o (31 \% change w.r.t. $E^{(10)}$ exch of HP-PH-pl). The relative change in the correlation contribution $E_{\text {disp }}^{(2)}$ is smallest. It changes only by $24 \%\left(-37.5 \mathrm{~kJ} \mathrm{~mol}^{-1}\right.$ for HP-PH- $p l$ and $-46.5 \mathrm{~kJ} \mathrm{~mol}^{-1}$ for HP-PH-o). Based on this brief comparison a correlation of the strength of the interaction and the magnitude of the SAPT contributions can be assumed. However, the quality of the basis set and the fact that only second order contributions were evaluated allow no deeper insight into the systems interaction. The values are to be considered to be of rather qualitative than quantitative nature.

\section{Conclusions}

The comparison of the interaction energies of $\pi$ sandwich structures of benzene - benzene and benzene - hexafluorobenzene dimers computed by our approach (see Computational details) with other theoretical calculations leads to the conclusion, that the methodology is applicable. Thus it was applied in the computational study of the sandwich structures of pyridine and pentafluoropyridine dimers which is the scope of this work.

From the presented results it can be concluded that the stabilization energy of the studied dimers depends on the relative positions of the monomers. Such behavior was surely anticipated. Evidently, the most favored structures are those with dominant Coulomb interactions such as in the case of $\mathrm{N}-\mathrm{H}$ interactions. Therefore, the strongest dependence of the stabilization energy on the relative orientation can be observed in HP-PH dimers. A relatively modest dependence in the HP-PF dimers can be attributed to an analogous effect, where the $\mathrm{F}-\mathrm{H}$ interaction is relatively available in any orientation. In the FP-PF dimers the interplay between the repulsive $\mathrm{F}-\mathrm{F}$ and $\mathrm{N}-\mathrm{N}$ and attractive $\mathrm{F}-\mathrm{C}$ or C-C governs the optimal geometry. The presented results supply some starting points for a more detailed study with methods that provide more accurate stabilization energies (CCSD $(\mathrm{T}))$ and maybe a SAPT analysis to higher order perturbations. The effect of substituent may be an interesting subject to study, namely with respect to the structures abundant in biological systems. Comparative studies were presented by Lee and coworkers (Lee et. al. 2007) and Bachorz et al. (2008). This studies however present different sets of molecules than this work.

\section{Acknowledgement}

This work has been supported by the Scientific Grant Agency VEGA (contracts Nos. 1/0327/12, 1/07535/13 and 1/1072/11). We are grateful to the HPC center at the Slovak University of Technology in Bratislava, which is a part of the Slovak Infrastructure of High Performance Computing (SIVVP project, ITMS code 26230120002, funded by the European region development funds, ERDF) for the computational time and resources made available.

\section{References}

Bachorz RA, Bischoff FA, Höfener S, Kloper W, Ottiger P, Leist R, Frey JA, Leutwyler S (2008) Phys. Chem. Chem. Phys. 10: 2758.

Berg JM, Tymoczko JL, Stryer L (2010) Biochemistry $7^{\text {th }}$ ed., W. H. Freeman, ISBN: 1429229365.

Besler BH, Merz Jr. KM, Kollman PA (1990) J. Comp. Chem. 11: 431.

Bukowski R, Cencek W, Jankowski P, Jeziorska M, Jeziorski B, Kucharski SA, Lotrich VF, Misquitta AJ, Moszynski R, Patkowski K, Podeszwa R, Rybak S, Szalewicz K, Williams HL, Wheatley RJ, Wormer PES, Zuchowski PS; Part of SAPT suite. Version 2008.2.

Dunning TH, Jr. (1989) J. Chem. Phys. 98: 1007.

Frisch MJ, Trucks GW, Schlegel HB, Scuseria GE, Robb MA, Cheeseman JR, Montgomery JA, Jr., Vreven T, Kudin KN, Burant JC, Millam JM, Iyengar SS, Tomasi J, Barone V, Mennucci B, Cosi M, Scalmani G, Rega N, Petersson GA, Nakatsuji H, Hada M, Ehara M, Toyota K, Fukuda R, Hasegava J, Ishida M, Nakajima T, Honda Y, Kitao O, Nakai H, Klene M, Li X, Knox JE, Hratchian HP, Cross JB, Adamo C, Jaramillo J, Gomperts R, Stratmann RE, Yazyev O, Austin AJ, Cammi R, Pomelli C, Ochterski JW, Ayala PY, Morokuma K, Voth GA, Salvador P, Dannenberg JJ, Zakrzewski VG, Dapprich S, Daniels AD, Strain M-C, Farkas O, Malick DK, Rabuck AD, Raghavachari K, Foresman JB, Ortiz JV, Cui Q, Baboul AG, Clifford S, Cioslowski J, Stefanov BB, Liu G, Liashenko A, Piskorz P, Komaromi I, Martin RL, Fox DJ, Keith T, Al-Laham MA, Peng CY, Nakaryakkara A, Chalacombe M, Gill PMW, Johnson B, Chen W, Wong MW, Gonzales C, Pople JA (2003) GAUSSIAN 03. Revision A.1. Pittsburg. Pa.

Gung BW, Amicangelo JC (2006) J. Org. Chem. 71: 9261.

Hunter CA, Lawson KR, Perkins J, Urch CJ (2001) J. Chem. Soc. Perkin. Transl. 2: 651.

Krishnan R, Binkley JS, Seeger R, Pople JA (1980) J. Chem. Phys. 72: 650.

Lee EC, Kim D, Jurečka P, Tarakeshwar P, Hobza P, Kim KS (2007) J. Phys. Chem. 111: 3446.

Meyer EA, Castellano RK, Diederich F (2003) Angew. Chem. Int. Ed. 42: 1210.

Møller C, Plesset MS (1934) Phys. Rev. 46: 618. 
Pitoňák M, Neogrády P, Rezáč J, Jurečka P, Urban M, Hobza P (2008) J. Chem. Theory. Comput. 4: 1829.

Pitoňák M, Riley KE, Neogrády, Hobza P (2008/a) Chem. Phys. Chem. 9: 1636.

Singh UC, Kollman PA (1984) J. Comp. Chem. 5: 129.

Tsuzuki S, Uchimaru T, Mikami M (2006) J. Phys. Chem. 110: 2027.
Tsuzuki S, Uchimaru T, Matsurama K, Mikami M, Tanabe K (2000) Chem. Phys. Lett. 319: 547.

Woon DE, Dunning TH, Jr. (1993) J. Chem. Phys. 98: 1358.

Xantheas SS (1996) J. Chem. Phys. 104: 8821. 